\title{
ESTUDO DO MANEJO DO ESTRESSE EM PACIENTES ACOMETIDOS POR ALOPECIA AREATA
}

\author{
Latife Yazigi \\ Sérgio Baxter Andreoli \\ Simone Maria Godinho"
}

\begin{abstract}
RESUMO. Alopecia Areata é uma dermatose caracterizada pela perda repentina de pêlos em uma única ou em várias áreas, na forma de círculo. Normalmente a perda se inicia no couro cabeludo, podendo também ocorrer em outras partes do corpo. Fatores emocionais traumáticos são referidos como desencadeantes. Um grupo de 12 pacientes acometidos de alopecia areata foi avaliado por meio de entrevista psicológica e do método de Rorschach (Sistema Compreensivo por Exner), com vista a identificar as condições de personalidade dos pacientes no que se refere ao manejo do estresse. Todos os pacientes relataram episódios traumáticos em suas vidas que poderiam ser considerados como desencadeantes da doença, e nove apresentaram nítidas dificuldades no controle e manejo do estresse.
\end{abstract}

Palavras-chave: alopecia areata, fatores estressantes, método de Rorschach.

\section{A STUDY OF STRESS MANAGEMENT IN PATIENTS STRICKEN BY ALOPECIA AREATA}

\begin{abstract}
Alopecia areata is a common dermatological disorder characterized by the rapid loss of hair in single or multiple patches. Hair loss is often on the scalp, but can also occur in other areas of the body. Psychological traumas have often been cited as precipitating factors. An evaluation of a group of twelve patients diagnosed with alopecia areata was undertaken using psychological interviews and the Rorschach Test, Comprehensive System, with the aim being to identify the condition of the patients' personalities while handling stress. All patients reported having experienced traumatic events which can be considered catalysts of alopecia areata symptoms, and nine demonstrated clear evidence of serious difficulty in stress management.
\end{abstract}

Key words: Alopecia Areata, stress factor, Rorschach for the Comprehensive System.

\section{ESTUDIO DEL MANEJO DEL ESTRÉS EN PACIENTES ACOMETIDOS POR ALOPECIA AREATA}

\begin{abstract}
RESUMEN. Alopecia Areata es una dermatosis caracterizada por la pierda de pelos, de forma rápida y en áreas. La pierda ocurre normalmente en los cabellos, como también en partes del cuerpo. Factores emocionales traumáticos son referidos como desencadenantes. Un grupo de 12 pacientes acometidos de alopecia areata fue evaluado por medio de entrevista psicológica y método de Rorschach (Sistema Comprensivo por Exner), pretendiendo un estudio exploratorio de la dinámica emocional y de la influencia de los factores psicológicos en la etiología de la alopecia areata. Todos los pacientes relataron episodios traumáticos en sus vidas que podrían ser considerados como desencadenantes de la enfermedad, y nueve presentaron nítidas dificultades en el control y manejo del estrés.
\end{abstract}

Palabras-clave: Alopecia, estresante, metodo Rorschach..

Alopecia Areata é uma doença dermatológica que resulta na perda de pêlos e cabelos, de forma e extensão variáveis (Sampaio, 1978). É uma doença crônica cuja manifestação clínica fundamental é a

* Doutora em Psicologia. Professora Titular do Departamento de Psiquiatria da Universidade Federal de São Paulo.

\# Mestre e Doutor em Medicina. Professor Assistente na Universidade Católica de Santos e Professor Afiliado da Universidade Federal de São Paulo.

Il Mestre em Ciências da Saúde. Psicóloga voluntária do Departamento de Psiquiatria da Universidade Federal de São Paulo. 
queda de pêlos, localizada ou generalizada, sem sinais inflamatórios ou de atrofia.

Segundo Fitzpatrick (1993), são quatro os tipos de Alopecia Areata: (a) alopecia em áreas, com perda de cabelo em uma ou mais regiões do couro cabeludo; podendo se estender a outras regiões pilosas do corpo; (b) alopecia total, que compromete todo ou quase todo o couro cabeludo; (c) alopecia universal, que compromete todas as áreas pilosas do corpo; (d) alopecia ofiásica, que compromete a área marginal do couro cabeludo.

A característica inicial da lesão da Alopecia Areata é uma perda delimitada de cabelo, normalmente notada por parentes, amigos ou cabeleireiros. Em $60 \%$ dos casos, o couro cabeludo é a primeira área afetada; em outros, a queda de pêlos pode se dar nas sobrancelhas, nos cílios ou em outras áreas do corpo, inclusive as genitais (Sampaio, 1978; Rook, 1992; Fitzpatrick, 1993).

Após o aparecimento da primeira lesão, a evolução da Alopecia Areata se dá de maneira variável, observando-se casos de um único episódio de perda em pequenas áreas com repilação espontânea até casos de perda total dos pêlos em questão de dias. Ocorrendo uma perda discreta, a repilação pode se dar em poucos meses ou podem surgir novas lesões após um intervalo de três a seis semanas. Em outros casos, a perda inicial do cabelo pode ser difusa, levando a uma perda total dentro de um período de 48 horas. Casos existem em que esta perda pode continuar difusa, não chegando a ser total. Esta situação instável entre queda e repilação pode durar anos. Relatos de caso mostram pacientes com até 20 anos de história da doença, caracterizando a Alopecia Areata como uma doença crônica. Os fios de cabelo, quando renascem, apresentam-se finos e despigmentados, porém com o decorrer do tempo evoluem para calibre e cor normais (Dudley, 1979; Fitzpatrick, 1993).

As estimativas sugerem que cerca de 1,7\% da população geral apresente pelo menos um episódio de Alopecia Areata durante a vida (Safavi et al., 1995) e ambos os sexos são afetados de modo semelhante (Fitzpatrick, 1993). Não há nenhum estudo sobre a incidência dessa doença na população brasileira.

O prognóstico da Alopecia Areata varia de acordo com sua forma clínica. Assim, perdas em áreas esparsas podem evoluir favoravelmente, com repilação espontânea, podendo o mesmo ocorrer em casos de perda total dos cabelos, em período de dias (Fitzpatrick, 1993). Já as alopecias total, universal e ofiásica apresentam pior prognóstico, uma vez que respondem menos aos tratamentos conhecidos.
A etiologia e a fisiopatologia de alopecia areata ainda permanecem obscuras, mas sabe-se que é uma doença multifatorial com componentes auto-imunes, atuando em indivíduos geneticamente predispostos (Rivitti, 2005).

$\mathrm{O}$ estresse emocional tem sido freqüentemente citado como desencadeador da doença. Assim, episódios traumáticos agudos, como divórcio, luto e perda de emprego, podem provocar desequilíbrio psicológico com conseqüente queda de pêlos no indivíduo (Perini et al., 1984; Backer, 1987; Invernizzi et al., 1987; Colón et al., 1991). A relação entre as questões emocionais da personalidade e distúrbios dermatológicos tem sido um desafio para os médicos que buscam aprimorar a efetividade da terapia dermatológica, uma vez que os mecanismos psicológicos ainda não estão claramente definidos. A demonstração científica da participação de fenômenos psiquiátricos na gênese de Alopecia Areata é muito difícil. A possível explicação bioquímica dos mecanismos patogênicos provocados por condições emocionais estaria na produção de neuromediadores capazes de interferir na imunidade (Rivitti, 2005).

Estudos identificaram, por meio de observações clínicas e avaliações psicológicas, influência marcada de ansiedade, depressão e mesmo de excitação pelos distúrbios de pele. Além disso, este tipo de doença produz evidentes alterações psicológicas pelo prejuízo na auto-imagem, devendo-se considerar a possibilidade de os fenômenos emocionais interferirem na afecção e a própria enfermidade produzir alterações psicológicas importantes (Rivitti, 2005). No caso da Alopecia Areata, freqüentemente é citado o forte impacto na área emocional e social em razão da perda dos cabelos e/ou pêlos (Delamere, 2008).

Reinhold (1960) refere a existência de uma interrelação com a depressão. Giuliane e Gentili (1986) citam trabalhos em que os pacientes alopécicos são identificados ora como neuróticos, ora como borderlines, ora como psicóticos (Russel \& Wittkower, 1953; Greemberg, 1955; MacAlpin, 1958). Estudos revelaram que o efeito das doenças dermatológicas crônicas que afetam a imagem corporal, tais como Alopecia Areata, psoríase e acne, pode levar o paciente a pensamentos suicidas (Gupta, 1998). Além disso, autores identificaram distúrbios psiquiátricos em pacientes que apresentavam algum sintoma dermatológico crônico (Picardi, 2003) e relataram que a probabilidade de co-morbidades psiquiátricas em pacientes que não apresentaram melhora era três vezes mais alta do que nos que melhoraram. Em contrapartida, Paga et al. (1992) não 
encontraram em suas pesquisas presença de depressão ou de outros fatores emocionais, tampouco a influência de fatores estressantes de vida. Nos últimos anos, tem sido privilegiada a relação entre Alopecia Areata e alexitimia (Poot, 2004), termo sugerido por Sifneos (1973) ao se referir ao paciente com dificuldades em identificar seus próprios estados emocionais. Nessa categoria incluir-se-iam alguns pacientes psicossomáticos que definem as emoções em termos de sensações somáticas ou de reações comportamentais ao invés de relacioná-las a experiências mentais.

O enfoque psicossomático definido por Marty (1993) propõe a hipótese de um funcionamento atípico do aparelho psíquico em pacientes que desenvolvem doenças somáticas, como as auto-imunes. O autor também desenvolve o conceito de pensamento operatório caracterizado pela aparente ausência de vida mental, com fantasias, e freqüentemente associada a distúrbios somáticos. Outro conceito abordado por Marty foi o de depressão essencial. Esta sintomatologia depressiva define-se pela falta de expressão da vida mental e se estabelece quando acontecimentos traumáticos comprometem certo número de funções psíquicas, tais como identificações, condensações, deslocamentos, introjeções e projeções. Angústias difusas precedem a depressão essencial, provocando uma aflição profunda no indivíduo.

McDougall (1996) observou que alguns de seus pacientes não percebiam suas emoções em situações angustiantes. Nestes casos, as idéias que estavam associadas a qualquer afeto conflituoso eram imediatamente apagadas da consciência. Relata o autor que tais pacientes funcionavam psiquicamente como bebês, que, não podendo expressar seus pensamentos por palavras, só conseguiam fazê-lo por meio de expressão de fenômenos psicossomáticos. Esta seria uma forma imatura de personalidade, que acaba por dificultar a formação de uma identidade própria do indivíduo. Este fato é consequiência de uma falha nos cuidados primários no início de vida da criança.

Para Winnicott (1999), a identidade pessoal só se concretiza em cada indivíduo quando há uma maternagem satisfatória, o que só ocorre quando a mãe consegue funcionar como um agente adaptativo, apresentando o mundo ao bebê, aos poucos, de forma que lhe possibilite criar referenciais apropriados para lidar com a realidade.

As doenças somáticas decorrem, geralmente, das inadequações do indivíduo às condições de vida com que se depara. Como a vida jamais se apresenta nas condições almejadas, as pessoas devem se adaptar a elas da melhor maneira possível e com os recursos internos de que dispõem. Quando o indivíduo não se encontra adaptado emocionalmente às situações de vida, é o aparelho somático que responde. O efeito desestabilizador nestas situações é o trauma. Esta dinâmica mental se assemelha aos pacientes alopécicos, que muitas vezes associam a queda de cabelo a algum fator estressante ocorrido em suas vidas.

É importante ressaltar que alguns estudos recentes sobre alopecia areata e aspectos emocionais consideram o estresse, a depressão e a ansiedade como participantes na etiologia da doença associados à alta prevalência de características de alexitimia (Cordan, 2006). Já outros estudos (Güleç, 2004) comentam ser pouco provável que a ansiedade e a depressão tenham papel importante na etiologia da doença, mas que o estresse é, de fato, desencadeante importante, causando um impacto negativo na qualidade de vida do paciente. A divergência destes estudos revela a dificuldade dos profissionais da área da saúde em explicar a Alopecia Areata e encontrar soluções mais eficazes para seu tratamento. É necessário que mais estudos sejam realizados tendo como objetivo a qualidade de vida do paciente (Delamere, 2008).

Kakourou (2007), em seu estudo com crianças, focalizou somente os aspectos clínicos da doença, uma vez que não há suficientes estudos epidemiológicos com estes pacientes. Outros estudos (Goh, 2007) se preocuparam com o custo da doença e a necessidade de se formarem grupos de apoio, com o objetivo de informar pacientes e familiares sobre novas medicações e opções de tratamento, assim como,possibilitar a troca de experiências pessoais.

\section{OBJETIVO}

O presente estudo tem como objetivo identificar as condições de personalidade dos pacientes com Alopecia Areata no que se refere ao manejo do estresse.

\section{MÉTODO}

O estudo é de caráter observacional em relação à dinâmica emocional dos pacientes com Alopecia Areata. Seu delineamento é de relato de caso que pode envolver descrições detalhadas de um ou de mais casos, segundo Fletcher (1996). Este autor comenta que aproximadamente 20 a $30 \%$ dos artigos originais, publicados em revistas médicas, são estudos de caso 
com limite em torno de dez pacientes tratando de maneira importante as doenças novas ou desconhecidas, objeto do presente estudo. Essa estratégia de relato de caso utiliza a abordagem tradicional de pesquisa qualitativa na compreensão dos fenômenos psíquicos (Martins, 1989).

\section{Participantes}

Um grupo de 12 pacientes com diagnóstico de alopecia areata do Ambulatório do Departamento de Dermatologia do Hospital São Paulo da Unifesp, foi selecionado segundo os seguintes critérios de inclusão: pessoas com (a) diagnóstico de alopecia areata em qualquer nível, universal, ofiásica, ou total; (b) em tratamento com difenciprone ou fazendo uso de corticóides de acordo com orientação médica; (c) idade variando de 15 a 50 anos; ambos os sexos, com qualquer tempo de história da doença. Estes critérios amplos foram estabelecidos dada a dificuldade da condição de cronicidade da doença. O percurso da Alopecia Areata não é previsível, podendo o paciente sofrer variações com diversos períodos de perda de cabelo e repilação durante a sua vida. Em muitos casos ocorre repilação em uma área e queda em outra. Isto acontece ao mesmo tempo e no mesmo paciente, o que dificulta definir o tipo de alopecia e o tempo de percurso da doença.

Os critérios de exclusão foram pessoas (a) usuárias de drogas; (b) com diagnóstico psiquiátrico ou em tratamento psicológico; (c) com idade inferior a 15 ou superior a 50 anos. Estes critérios foram assim determinados porque estes itens poderiam ser um viés nos resultados dos testes psicológicos.

O projeto foi aprovado pelo Comitê de Ética da instituição e todos os participantes concordaram com o estudo e assinaram o termo de consentimento livre e esclarecido.

\section{Instrumentos}

Os instrumentos selecionados foram a entrevista psicológica e o Método de Rorschach no Sistema Compressivo (Exner, 2003).

A entrevista psicológica foi utilizada para identificar a presença de eventos de vida traumáticos, estressantes ou angustiantes que poderiam ser considerados como desencadeadores dos sintomas de Alopecia Areata. Para isso a entrevistadora adotou a técnica de perguntas, e intervenções foram feitas quando se fizeram necessárias. O campo da entrevista se configurou de acordo com as características do entrevistado, porém com os limites preestabelecidos pelo entrevistador, ou seja: tempo, lugar e técnica (Bleger, 1979).
O Método de Rorschach foi utilizado para avaliar as condições de personalidade relativas ao manejo do estresse. Esse método tem se mostrado bastante eficiente como instrumento na área de investigação e diagnóstico da personalidade; e o Sistema Compreensivo tem garantido ao Rorschach o status de instrumento de grande confiabilidade, por sua objetividade e precisão nos resultados (Nascimento, 2002).

\section{RESULTADOS}

\section{Quanto à entrevista psicológica.}

Dos 52 pacientes encaminhados inicialmente para o estudo, somente 20 preencheram os critérios de inclusão; destes, quatro não aceitaram participar e quatro não compareceram. Dos 12 pacientes restantes avaliados, oito eram mulheres e quatro eram homens. No grupo das mulheres, uma paciente era casada, uma era divorciada e as demais eram solteiras. Das oito mulheres, somente duas trabalhavam. No grupo dos homens todos eram solteiros e trabalhavam ou estavam envolvidos em alguma atividade.

Todos os pacientes eram previdenciários e pertencentes à classe social baixa.

Das oito mulheres, cinco apresentaram alopecia universal e três, alopecia total. Entre os homens, um apresentou alopecia universal e três apresentaram alopecia total. Todos iniciaram o tratamento médico imediatamente após a manifestação da doença e não apresentavam co-morbidades clínicas. O tempo de história da doença variou entre três e 16 anos.

De modo geral, os pacientes se mostraram interessados em um primeiro contato, apesar de se expressarem de maneira cautelosa e mesmo rígida, sendo econômicos nas palavras e nas informações.

Houve unanimidade nas impressões pessoais: todos se consideravam pessoas nervosas e gostavam de ficar sozinhos e calados. Relataram episódios traumáticos em suas vidas, que poderiam ser considerados desencadeantes da alopecia, como: morte do pai, cancelamento do próprio casamento, sarampo, gravidez da namorada, separação dos pais, estupro, agressão física de irmão alcoólatra e nascimento de outro membro da família.

Não foi possível verificar exatamente qual o período em que ocorreu o fator estressante antes do aparecimento da doença. Este fato é totalmente compreensível, dada a dificuldade dos pacientes em perceber suas emoções em situações angustiantes. 
Todos os pacientes comentaram já haver-se habituado à perda de cabelos e das sobrancelhas, ou até mesmo a vê-los renascer e cair após algum tempo.

Nenhum paciente relatou caso de Alopecia Areata na família. Embora nenhum deles tenha se mostrado esperançoso quanto à recuperação dos pêlos, todos relataram seguir à risca as instruções dadas pelos médicos e aceitaram participar do estudo já na primeira entrevista. Sempre retornaram, quando solicitados para eventuais esclarecimentos, sendo muito solícitos e colaboradores.

\section{Quanto ao Método de Rorschach, Sistema Compreensivo}

Dos 12 protocolos, nove apresentaram sinais positivos na constelação Controle e Manejo do Estresse, $C D I$, denominada Déficit de Relacionamento Interpessoal, que informa sobre as condições subjetivas da pessoa para fazer face às demandas internas e externas.

Com exceção de um, os pacientes apresentaram pelo menos três das condições que compõem o $C D I$, o que confirma a dificuldade para enfrentar situações estressantes. Destas condições, as mais freqüentes diziam respeito às variáveis $E A \quad(M+$ WSumC: respostas de movimento humano + soma ponderada das respostas à cor); $C O P$ (respostas de movimento em cooperação); $A G$ (repostas de movimento agressivo); $H$ (respostas de figura humana), $T$ (respostas de textura) e presença de $F d$ (respostas de alimento).

Os pacientes apresentaram carência de recursos internos disponíveis da ordem da constituição do self, da autonomia, da empatia e das manifestações afetivas. Revelaram dificuldade em atividades de parceria e de assertividade interpessoal. Os problemas nos processos identificatórios revelaram retração, inibição ou pouco interesse em relacionamentos, característica confirmada pela ausência de busca de contato com o outro. Permitiram identificar imaturidade, dependência e expectativa de que outros viessem a atender às suas necessidades e os ajudassem na solução de seus problemas.

É importante ressaltar que, segundo Weiner (2000), tomar decisões e resolver problemas constituem uma necessidade que as pessoas experimentam em seu cotidiano. Seja qual for a origem destas demandas - como pensamentos e sentimentos (eventos internos) e opiniões de outras pessoas (eventos externos) -, elas constituem um estresse; por isso, de algum modo, a pessoa deve ser atendida para sentir-se bem e comportar-se de modo eficaz. Quanto mais apropriada for a maneira como a pessoa atende às suas próprias necessidades, maior é a probabilidade de êxito no manejo do estresse e de obtenção de um bom ajustamento psicológico. Em contraposição, as dificuldades no atendimento às demandas da vida conduzem as pessoas ao fracasso no manejo do estresse. Este é o caso dos pacientes alopécicos.

Embora não seja o objetivo do presente estudo analisar as demais constelações, chama a atenção a presença do Índice de Percepção e Pensamento [PTI] e da Constelação de Suicídio [S-CON], que indicam a presença de outras problemáticas psicológicas que deverão ser investigadas futuramente. Faremos um breve comentário de algumas destas constelações.

A Constelação de Suicídio [S-CON] é um sinal de alerta, pois indica que a pessoa corre o risco de executar atitudes autodestrutivas. Dos 12 protocolos avaliados, sete pacientes demonstraram variáveis que indicam predisposição ao suicídio, com destaque para baixa auto-estima, tom pessimista, autocrítica negativa, dificuldades nas relações interpessoais e, principalmente, aumento da sobrecarga emocional interna e sérias dificuldades na modulação afetiva. Estas características favorecem as percepções distorcidas, gerando interferência na captação do que ocorre a sua volta.

O Índice de Percepção e Pensamento (PTI) informa-nos sobre problemas nos processos cognitivos de mediação e ideação. Quando se encontra em estado de confusão mental, freqüentemente a pessoa sofre prejuízos nestas funções. Dos três pacientes que apresentaram esta constelação, todos tiveram pontuação positiva nas cinco condições, o que significa sérias dificuldades cognitivas. Esta constelação indica carência de controle da impulsividade ideativa e maior incidência de relações implausíveis, desorganização ideativa e afetiva, afastamento da realidade em decorrência de falha no processo de julgamento e menor capacidade de manutenção do encadeamento do fluxo de pensamento.

\section{DISCUSSÃO DOS RESULTADOS}

A despeito do pequeno tamanho da amostra deste estudo, pode-se dizer que os pacientes alopécicos apresentaram características semelhantes àquelas de pacientes com sintomas somáticos e revelaram uma forma arcaica de funcionamento psíquico, uma vez que o indivíduo não emprega as palavras como veículo transmissor de seu pensamento, o que, segundo McDougall (1996), é característica dos pacientes ditos 'psicossomáticos'. Além disso, apresentaram 
características muito próximas da alexitimia: dificuldades em identificar e comunicar sentimentos, com tendência a evitar relacionamentos mais próximos. $\mathrm{O}$ aumento da tensão psíquica interfere de modo contraproducente na maneira de o paciente se organizar e direcionar seu comportamento. Este fato pode provocar vivências de desamparo e levá-lo a sentir-se indefeso quando solicitado em suas atividades cotidianas e sociais. Esta característica de organização mental revela personalidade mais imatura, vulnerabilidade diante de situações de tensão e prejuízo na esfera do controle nas suas relações interpessoais. Esta característica os aproxima do conceito de alexitimia de Sifneos (1975), deixando evidente a dificuldade em definir seus estados afetivos.

Segundo Winnicott (1990), o objetivo inconsciente da doença psicossomática é manter uma dissociação entre mente e corpo. Esta clivagem da personalidade tende a se aprofundar, o que torna o tratamento médico extremamente difícil. Para o autor, há na doença duas tendências: a da doença física e seus efeitos sobre o desenvolvimento psíquico e a da doença psíquica e seus efeitos sobre o desenvolvimento físico. A cisão seria a divisão entre o cuidado físico e a compreensão intelectual, ou seja, separação entre o soma e a psique. Essa clivagem mental do paciente é uma defesa contra o mundo externo, vivenciado como hostil e persecutório em sua fantasia.

O surgimento da Constelação de Suicídio e o Índice de Percepção e Pensamento sugerem déficits na regulação da emoção. A influência do estresse durante o tratamento da doença e as fortes repercussões estéticas no ambiente social podem levar o paciente a comportamentos fóbicos e impulsivos e a fortes crises de ansiedade. Além disso, estudos revelam que pacientes que não mostram sinais de melhora com o tratamento apresentam maior incidência de distúrbios psiquiátricos (Picardi et al., 2003).

Para melhor interpretação deste resultado, faz-se necessário o uso de escalas mais específicas quanto a problemas de pensamento, retraimento, ansiedade e depressão, o que pode auxiliar na elaboração de um perfil do grupo estudado.

Na prática clínica deve-se dar atenção ao impacto da doença sobre os membros da família. Estudos revelaram que psicoterapias individuais de curta duração e trabalho psicoterapêutico com famílias de pacientes alopécicos ajudam muito na qualidade de vida de todos os membros (Liakopoulou, 1997).

Outro ponto importante é a necessidade de haver programas envolvendo profissionais de diversas áreas
- neste caso, dermatologistas e psiquiatras (Picardi, 2003).

Brajac et al. (2003) mostraram em seus achados que pacientes alopécicos experimentam grande benefício com tratamentos psicológicos. Os mesmos autores apresentaram também tratamentos clínicos específicos para as suas lesões. Winnicott (1999) propõe que a variedade de psicoterapia deveria depender não do ponto de vista do terapeuta, e sim, da necessidade do paciente. Sugere, assim, modificações técnicas adequadas ao paciente e respectivo tratamento. Deste modo, o terapeuta conseguiria manejar a situação de forma a permitir ao paciente a compreensão de sua história de acordo com seu ritmo pessoal.

\section{CONCLUSÃO}

Quanto às condições de personalidade envolvidas no manejo do estresse $(C D I)$, podemos concluir que os pacientes deste estudo não apresentam recursos internos (afetivos e cognitivos) suficientes nem habilidades para utilizar os recursos internos disponíveis, do que resulta certa inadequação no controle de suas condutas.

Apesar da limitação da amostra, este estudo sugere que traços de personalidade, fatores sociais e dificuldades em se relacionar com outras pessoas podem estar relacionados com a etiologia da doença. Além disso, as características de alexitimia reveladas neste estudo podem ser consideradas como um fator de risco para a doença, pois a dificuldade em identificar e expressar verbalmente pensamentos e emoções faz com que o paciente se exponha cada vez mais a situações estressantes sem se dar conta disso.

É importante ressaltar que a pequena amostra dos pacientes limita as generalizações quanto aos resultados. Não obstante, os achados do presente estudo sinalizam a importância de futuras pesquisas dentro desta área com a utilização de outros instrumentos, o que poderia agregar informações e fundamentar atitudes terapêuticas mais abrangentes.

\section{REFERÊNCIAS}

Backer,G.H.B.(1987). Invited review: psychological factors and immunity. Journal of Psychosomatic Research, 31, 1-10.

Bleger,J.(1979). Temas de Psicologia- Entrevista y grupos (pp.09-19), Buenos Aires: Ediciones Nueva Visión.

Brajac, I.(2003).Roles of stress, stress perception and trait-anxiety in the onset and course of alopecia areata. The Journal of Dermatology, 30, 871-878. 
Colón, E.A. (1991). Lifetime prevalence of psychiatric disorders in patients with alopecia areata. Compr Psychiatric, 32: 245-251.

Cordan Yazici A.; Basterzi A.; Alopecia Areata and Alexithymia. Turk Psikiyatri Derg, 17(2): 101-6.

Delamere, F.M; Sladden, M.M., (2008). Interventions for alopecia areata. Cochrane Database Syst Ver, 16(2): CD004413.

Dudley, F. (1979). Diagnóstico diferencial (pp.17-21), Rio de Janeiro: Guanabara Koogan.

Exner, J. E. Jr. (2003). The Rorschach: A comprehensive system. New York: Wiley.

Fitzpatrick, T.B.; Eisen, A.Z.; Wolf, K.; Fredberg, I.M.; Austen, K.F.K. (1993.) Dermatology in General Medicine (vol I, pp. 671685), New York: Mcgraw-Hill.

Fletcher, R.H.(1996). Epidemiologia Clínica: Elementos Essenciais (pp.217-221). Porto Alegre: Artes Médicas.

Giuliani, M.; Gentili, G. (1986). Dermatosi psicosomatica e sviluppo de 22 casi di alopecia areata indagati com metodi projettivi. Med. Psicosom, 31:13-30.

Greenberg, S.J. (1955). Alopecia Areata: A psychiatric survey. American Medical Association Archieves of Dermatology 72: 454-457.

Goh, C.; Lane, A.T. (2007). Support groups for children and their families in pediatric dermatology. Pediatr Dermatol, 24(3): 302-5.

Güleç A.T.; Tanriverdi N. (2004). The role of psychological factors in alopecia areata and the impact of the disease on the quality of life. Int J Dermatol,43(5): 352-6.

Gupta,M.A.,Gupta A.K.(1998). Depression and suicidal ideation in dermatology patients with acne, alopecia areata, atopic dermatitis and psoriasis. Br J Dermatol,139(5): 846:850.

Invernizzi, G. (1987). Life events and personality factors in alopecia areata. Med Sci Res 15, 1219-1220.

Kakourou,T.; Karachristou K. (2007). A case series of alopecia areata in children: impact of personal and family history of stress and autoimmunity. J Eur Acad Dermatol Venerol, 21(3): 356-9.

Liakopoulou, M. (1997). Children with alopecia areata: psychiatric symptomatology and life events. J Am Acad Adolesc Psychiatry, 36(5): 678-84

MacAlpine,I.(1958). Is alopecia areata psychosomatic? A psychiatric study. British Journal of Dermatology 70, 117-131.

Martins J., Bicudo, M.A. (1989). A Pesquisa qualitativa em Psicologia: fundamentos e recursos básicos. São Paulo: Educ.

Marty, Pierre (1993). A Psicossomática do adulto. Porto Alegre: Artes Médicas.

McDougall, J. (1996). Teatros do Corpo: o Psicossoma em Psicanálise (pp.06-26). São Paulo: Martins Fontes.
Nascimento, Regina S.G.F. (2002). A normative research on the Rorschach comprehensive system in the city of São Paulo. PsicoUSF, 7(2): 127-141.

Paga G, Faina P, Teodori A, Angelini $C$, Biondi M, Ruvinetti S. (1992). Psychological factors in alopecia areata: results from preliminary, controlled study. New Trends Exp Clin Psychiatry 8, 73-79.

Perini GI, Veller Fornasa C, Cipriani R, Bettin A, Zecchino F., Peserico A.(1984). Life events and alopecia areata. Psychother Psychosom,41:48-52.

A. Picardi, Pasquini P., Cattaruzza M.S., Gaetano P., Baliva G., Melchi C.F., Papi M., Camaioni D., Tiago A., Gobello T., Biondi M. (2003). Psychosomatic factors in first-onset alopecia areata. Psychosomatics, 44: 374-381.

Poot, F. (2004). Psychological consequences of chronic hair diseases. Rev. Med Brux,25: A286-288.

Reinhold, M.( 1960). Relationship of stress to the development of symptoms in Alopecia Areata and chronic urticaria. British Medical Journal, 1,846-849.

Rivitti, E. A. (2005). Alopecia Areata: Revisão e atualização. An Bras Dermatol, 80(1), 57-68.

Rook, Wilkinson, Ebling.(1992). Textbook of dermatology (pp.25862594). Oxford:Blackwell.

Russel, B.; Wittkower, E. ( 1953). Emotional factors in skin diseases (pp.176).New York: Harper\& Brothers.

Safavi, K. (1950). Incidence of alopecia areata in Olmested County, Minnesota, 1975 trough 1989. Mayo Clin Proc. 70, 628-33.

Sampaio, S. (1978). Dermatologia Básica.(pp.149-154). Porto Alegre: Artes Médicas.

Sifneos,P.(1973). The prevalence of alexithymic characteristics in psychosomatic patients. Psychoter Psychosom 22, 255-262.

Sifneos,P.(1975). Problems of psychotherapy in patients with alexithymic characteristics and physical disease, in Psychotherapy and Psychosomatics, 26; 65-70.

Weiner, I. B. (2000). Princípios da Interpretação do Rorschach. São Paulo: Casa do Psicólogo.

Winnicott, D.W. (1990). Natureza Humana. (pp.29-34). Rio de Janeiro: Imago.

Winnicott, D.W. (1999). Tudo Começa em Casa. (pp.13; 93-103). São Paulo: Martins Fontes.

Recebido em 28/08/2007 Aceito em 08/07/2008

Endereço para correspondência : Simone M. Godinho. Rua Buarque, 64, Morumbi-CEP 05618-060, São Paulo -SP, Brasil. E-mail: simonegodinho@osite.com.br 